\title{
An Analytic Characterization of a Stabilizing Feedback for LTI Plants
}

\author{
Andrés A. Peters, Diego A. Oyarzún, Eduardo I. Silva and Mario E. Salgado
}

\begin{abstract}
The characterization of all stabilizing controllers via the Youla Parameterization requires prior knowledge of one stabilizing feedback. This task is trivial in the case of stable plants. In the unstable case, one needs to use a suitable design technique to obtain such a stabilizing controller. The resulting controller is usually not an explicit function of plant dynamical features. In this paper, we propose a stabilizing controller design such that the sensitivity function can be expressed as an explicit function of the non-minimum phase zeros, time delays, and unstable poles of the plant (and their directions in the multiple-input multiple-output case). These dynamical features are known to impose fundamental limitations on control performance. The results in this paper highlight their relevance since they are shown to be the minimum information required to build a stabilizing controller.
\end{abstract}

Index Terms-Discrete-time LTI systems, Youla Parameterization, Stabilization.

\section{INTRODUCTION}

The basic control design problem consists of finding a feedback control law that stabilizes the plant and satisfies a number of design objectives. These control objectives include time and frequency domain requirements that must be taken into account at the design stage. In the case of linear timeinvariant (LTI) plants, there exists a vast range of available design methods [1], [2], [3]. Among them, optimal control strategies have been particularly popular. The success of such methods relies on the ability to appropriately parameterize the set of acceptable controllers in terms of the variable(s) to be optimized.

In this context, the well-known Youla Parameterization (YP; see, e.g., [4], [5]) provides, for a given LTI plant, a simple characterization of the class of all stabilizing controllers. This parametrization yields closed loop sensitivity functions that are affine in a parameter which is only required to be stable and proper. This allows to recast the optimization over the set of stabilizing controllers as a search over the set of stable and proper transfer matrices. The YP has been widely used in the solution of different optimal control problems such as, e.g., $\mathcal{H}_{2}$ and $\mathcal{H}_{\infty}$ optimal sensitivity problems [6], [7], [8], [9], and also mixed $\mathcal{H}_{2} / \mathcal{H}_{\infty}$ problems [10]. The original formulation of the $\mathrm{YP}$ is for rational plants, but recent extensions also allow for the incorporation of time delays [11].

In the discrete-time case, a simple reinterpretation of the YP was made in [12], and used to compute $\mathcal{H}_{2}$ performance

A. Peters, E. Silva and M. Salgado are with the Department of Electronic Engineering, Universidad Técnica Federico Santa María, Valparaiso, Chile apeters@elo.utfsm.cl, eduardo.silva@usm.cl, mario.salgado@usm.cl

D. Oyarzún is with the Hamilton Institute, National University of Ireland Maynooth, Ireland diego.oyarzun@nuim.ie bounds with closed loop pole location constraints [13]. The key aspect of this re-writing of the YP is that it makes immediately obvious which plant dynamical features limit performance for a given quadratic functional. Indeed, its construction is based on the description of the set of admissible sensitivity functions in terms of both finite and infinite non-minimum phase zeros, unstable plant poles (and their directions in the multiple-input multiple-output (MIMO) case), and the sensitivity achieved by an initial stabilizing controller design.

Regardless of which formulation is employed, the characterization of stabilizing controllers (equivalently, of admissible sensitivity functions) requires the prior knowledge of one stabilizing design. For stable plants such stabilizing controller can be simply set to zero, but in the unstable case a preliminary design stage is usually mandatory. Since this controller is not required to meet any performance specifications, any stabilizing design would fit the purpose. On the other hand, it is clear that optimal controllers are independent of the initial stabilizing controller choice. As a consequence, a natural question arises: what are the essential features of such an initial controller choice?

In this paper we tackle the problem of finding an explicit initial stabilizing design for controller parametrization. In particular, it is shown that an admissible sensitivity function can be built from the sole knowledge of the finite and infinite non-minimum phase zeros and unstable poles of the plant (and their directions in the MIMO case). Our results are valid for square rational MIMO discrete-time systems with simple unstable poles, and are given as an explicit closed form formula for such admissible sensitivity function in terms of plant dynamical features.

It is well known that non-minimum phase zeros and unstable poles impose fundamental limitations on closed loop performance [14]. The results presented in this paper further highlight their relevance, as they are shown to correspond to the minimum information required to build a stabilizing controller for a given plant. Additionally, the expressions provided are well suited for the development of explicit formulae for the best achievable performance, and the corresponding optimal controllers, when $\mathcal{H}_{2}$ or $\mathcal{H}_{\infty}$ measures are employed (see, e.g., [15], [16]).

The paper is organized as follows: Section II presents the main definitions and notation used throughout the paper. The YP and its alternative formulation is described in Section III. The main results of the paper are reported in Section IV. Illustrative examples and concluding remarks are included in Sections V and VI, respectively. 


\section{NOTATION, DEFINITIONS AND BACKGROUND MATERIAL}

We denote the set of $n \times n$ real rational discrete-time transfer matrices in the complex variable $z$ by $\mathcal{R}$, and the set of real rational stable and proper discrete-time transfer matrices by $\mathcal{R} \mathcal{H}_{\infty} \subset \mathcal{R}$. The transfer matrix $\boldsymbol{A}(z) \in \mathcal{R}$ has a (transmission) zero at $z=c \in \mathbb{C}$ if and only if the rank of $\boldsymbol{A}(c)$ is smaller than the normal rank of $\boldsymbol{A}(z)$ [17]. The left and right directions associated with a zero are $\boldsymbol{\eta} \in \mathbb{R}^{n}, \boldsymbol{\mu} \in \mathbb{R}^{n}$ such that $\boldsymbol{\eta}^{H} \boldsymbol{A}(c)=0$ and $\boldsymbol{A}(c) \boldsymbol{\mu}=0$ with $\boldsymbol{\eta}^{H} \boldsymbol{\eta}=\boldsymbol{\mu}^{H} \boldsymbol{\mu}=1$, respectively. If $|c| \geq 1,{ }^{1}$ the zero is called non-minimum phase (NMP), otherwise, is called minimum phase (MP). Similarly, a transfer matrix having at least one NMP zero is called NMP, otherwise, it is called MP.

Given a nonsingular almost everywhere (a.e.) transfer matrix $\boldsymbol{A}(z) \in \mathcal{R} \mathcal{H}_{\infty}$, we define the left unitary interactor (LUI) $\boldsymbol{E}_{\boldsymbol{L}}(z) \in \mathcal{R}$ of $\boldsymbol{A}(z)$ as a function $\boldsymbol{E}_{\boldsymbol{L}}(z)$ that is nonsingular a.e., has no finite NMP zeros, satisfies $\boldsymbol{E}_{\boldsymbol{L}}\left(z^{-1}\right)^{T} \boldsymbol{E}_{\boldsymbol{L}}(z)=$ $I$ and $\boldsymbol{E}_{\boldsymbol{L}}(1)=\boldsymbol{I}$, and is such that the transfer matrix $\boldsymbol{E}_{\boldsymbol{L}}(z) \boldsymbol{A}(z)$ is stable and MP (hence, also biproper). Similarly, we define the right unitary interactor (RUI) $\boldsymbol{E}_{\boldsymbol{R}}(z) \in$ $\mathcal{R}$ as a function such that $\boldsymbol{E}_{\boldsymbol{R}}(z)$ is nonsingular a.e., has no finite NMP zeros, satisfies $\boldsymbol{E}_{\boldsymbol{R}}\left(z^{-1}\right)^{T} \boldsymbol{E}_{\boldsymbol{R}}(z)=I$ and $\boldsymbol{E}_{\boldsymbol{R}}(1)=\boldsymbol{I}$, and is such that the transfer matrix $\boldsymbol{A}(z) \boldsymbol{E}_{\boldsymbol{R}}(z)$ is stable and MP. It can be shown that a RUI for $\boldsymbol{A}(z)$ is the transpose of a LUI for $\boldsymbol{A}(z)^{T}$, and vice versa. If $\boldsymbol{A}(z) \in \mathcal{R H}_{\infty}$ is non-singular, then unitary interactor matrices exist if and only if $\boldsymbol{A}(z)$ does not have zeros on the unit circle [18]. Denote the multi-set ${ }^{2}$ of NMP zeros of $\boldsymbol{A}(z)$ by $\left\{c_{1}, c_{2}, \ldots, c_{n}\right\}$. A LUI for $\boldsymbol{A}(z)$ is given by

$$
\boldsymbol{E}_{\boldsymbol{L}}(z)=\prod_{i=1}^{n} \boldsymbol{L}_{\boldsymbol{n - i + 1}}(z)
$$

where

$$
\begin{aligned}
& \boldsymbol{L}_{\boldsymbol{i}}(z) \triangleq\left(f_{i}(z)-1\right) \boldsymbol{\eta}_{\boldsymbol{i}} \boldsymbol{\eta}_{\boldsymbol{i}}{ }^{H}+I, \\
& f_{i}(z) \triangleq\left\{\begin{array}{ll}
\frac{\left(1-z \bar{c}_{i}\right)\left(1-c_{i}\right)}{\left(z-c_{i}\right)\left(1-\bar{c}_{i}\right)} & \text { if }\left|c_{i}\right|<\infty \\
z & \text { if } c_{i}=\infty
\end{array},\right.
\end{aligned}
$$

and $\boldsymbol{\eta}_{\boldsymbol{i}} \in \mathbb{R}^{n}$ is such that $\boldsymbol{\eta}_{\boldsymbol{i}}{ }^{H} \hat{\boldsymbol{A}}_{\boldsymbol{i}}\left(c_{\boldsymbol{i}}\right)=0$, with

$$
\begin{aligned}
\hat{\boldsymbol{A}}_{\boldsymbol{i}+\mathbf{1}}(z) & \triangleq \boldsymbol{L}_{\boldsymbol{i}}(z) \hat{\boldsymbol{A}}_{\boldsymbol{i}}(z), \quad i=1,2, \cdots, n, \\
\hat{\boldsymbol{A}}_{\mathbf{1}}(z) & \triangleq \boldsymbol{A}(z) .
\end{aligned}
$$

In this paper we consider the standard one degree of freedom control loop depicted in Figure 1, where the plant $\boldsymbol{G}(z)$ satisfies the following:

Assumption 1: $\boldsymbol{G}(z) \in \mathcal{R}$ is nonsingular a.e., is proper, has no poles or zeros on the unit circle, and has no NMP zero at the same location of an unstable pole. Moreover, every unstable pole of $\boldsymbol{G}(z)$ has multiplicity equal to one.

\footnotetext{
${ }^{1}$ This definition encompasses zeros at infinity (associated with time delays).

${ }^{2}$ Multi-sets are "sets" where multiplicity matters (see, e.g., [19]).
}

The general case, i.e. a plant with unstable poles of arbitrary multiplicity is omitted due to space limitations but the ideas described here apply to it in a similar fashion. Since $\boldsymbol{G}(z)$ is real rational, it admits coprime matrix fraction descriptions in $\mathcal{R H}_{\infty}$ given by [20]

$$
\boldsymbol{G}(z)=\boldsymbol{D}_{\boldsymbol{L}}(z)^{-1} \boldsymbol{N}_{\boldsymbol{L}}(z)=\boldsymbol{N}_{\boldsymbol{R}}(z) \boldsymbol{D}_{\boldsymbol{R}}(z)^{-1},
$$

where $\boldsymbol{D}_{\boldsymbol{L}}(z), \boldsymbol{N}_{\boldsymbol{L}}(z), \boldsymbol{N}_{\boldsymbol{R}}(z)$ and $\boldsymbol{D}_{\boldsymbol{R}}(z)$ are all in $\mathcal{R} \mathcal{H}_{\infty}$. Every NMP zero of $\boldsymbol{G}(z)$ is also a zero of $\boldsymbol{N}_{\boldsymbol{R}}(z)$ with the same algebraic and geometric multiplicity [21]. Similarly, every unstable pole of $\boldsymbol{G}(z)$ is a NMP zero of $\boldsymbol{D}_{\boldsymbol{L}}(z)$. We define the zero interactor of $\boldsymbol{G}(z)$, denoted by $\boldsymbol{E}_{\boldsymbol{c}}(z)$, as the LUI of $\boldsymbol{N}_{\boldsymbol{R}}(z)$. Analogously, we define the pole interactor of $\boldsymbol{G}(z)$, denoted by $\boldsymbol{E}_{\boldsymbol{p}}(z)$, as the RUI of $\boldsymbol{D}_{\boldsymbol{L}}(z)$. From the definition of interactor matrices, it follows that $\boldsymbol{E}_{\boldsymbol{c}}(z) \boldsymbol{G}(z)$ is MP and biproper, whereas $\boldsymbol{E}_{\boldsymbol{p}}(z)^{-1} \boldsymbol{G}(z)$ is a stable transfer matrix.

A controller $\boldsymbol{C}(z) \in \mathcal{R}$ is called admissible for $\boldsymbol{G}(z)$ if and only if it yields an internally stable and well-posed feedback loop [22]. We refer to an admissible loop (or design) as one that is generated by an admissible controller. From Figure 1, it follows that the complementary sensitivity function, i.e., the transfer function between the reference signal $r$ and the plant output $y$, is given by

$$
\boldsymbol{T}(z) \triangleq \boldsymbol{G}(z) \boldsymbol{C}(z)(\boldsymbol{I}+\boldsymbol{G}(z) \boldsymbol{C}(z))^{-1} .
$$

By extension, the complementary sensitivity function of an admissible feedback loop is called admissible.

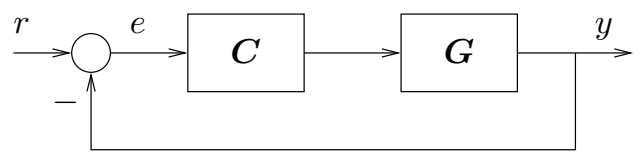

Fig. 1. One degree of freedom control loop.

\section{AFFINE PARAMETERIZATIONS OF ADMISSIBLE LOOPS}

\section{A. Classical Youla Parameterization (YP)}

In this section we briefly review the YP [23], [4], [5]. We consider the control configuration of Figure 1 and assume that an admissible controller $\boldsymbol{C}_{\boldsymbol{s}}(z)$ is available. Let

$$
\boldsymbol{C}_{\boldsymbol{s}}(z)=\boldsymbol{N}_{\boldsymbol{R} C}(z) \boldsymbol{D}_{\boldsymbol{R} C}^{-1}(z)=\boldsymbol{D}_{\boldsymbol{L C}}^{-1}(z) \boldsymbol{N}_{\boldsymbol{L C}}(z),
$$

be a right and left coprime factorization of $\boldsymbol{C}_{\boldsymbol{s}}(z)$, respectively. Then, every admissible controller $C(z)$ can be written as

$$
\begin{aligned}
& C(z)= \\
& \quad\left(\boldsymbol{N}_{\boldsymbol{R} C}(z)+\boldsymbol{D}_{\boldsymbol{R}}(z) \boldsymbol{Q}(z)\right)\left(\boldsymbol{D}_{\boldsymbol{R} C}(z)-\boldsymbol{N}_{\boldsymbol{R}}(z) \boldsymbol{Q}(z)\right)^{-1}
\end{aligned}
$$

where $\boldsymbol{Q}(z) \in \mathcal{R} \mathcal{H}_{\infty}$ is such that

$$
\operatorname{det}\left\{\boldsymbol{D}_{\boldsymbol{R} \boldsymbol{C}}(z)-\boldsymbol{N}_{\boldsymbol{R}}(z) \boldsymbol{Q}(z)\right\} \neq 0,
$$


$\boldsymbol{N}_{\boldsymbol{L}}(z), \boldsymbol{D}_{\boldsymbol{L}}(z), \boldsymbol{N}_{\boldsymbol{R}}(z)$ and $\boldsymbol{D}_{\boldsymbol{R}}(z)$ are defined as before, and

$$
\left[\begin{array}{cc}
\boldsymbol{D}_{\boldsymbol{L C}}(z) & \boldsymbol{N}_{\boldsymbol{L C}}(z) \\
-\boldsymbol{N}_{\boldsymbol{L}}(z) & \boldsymbol{D}_{\boldsymbol{L}}(z)
\end{array}\right]\left[\begin{array}{cc}
\boldsymbol{D}_{\boldsymbol{R}}(z) & -\boldsymbol{N}_{\boldsymbol{R} C}(z) \\
\boldsymbol{N}_{\boldsymbol{R}}(z) & \boldsymbol{D}_{\boldsymbol{R} C}(z)
\end{array}\right]=\boldsymbol{I}
$$

is satisfied.

This parameterization yields a complementary sensitivity function that is affine in $\boldsymbol{Q}(z)$. Indeed, it is easy to see that

$$
\boldsymbol{T}(z)=\boldsymbol{N}_{\boldsymbol{R}}(z)\left(\boldsymbol{N}_{\boldsymbol{L} C}(z)+\boldsymbol{Q}(z) \boldsymbol{D}_{\boldsymbol{L}}(z)\right) .
$$

Moreover, if $\boldsymbol{G}(z)$ is stable and $\boldsymbol{C}_{\boldsymbol{s}}(z)=0$, then (12) yields

$$
\boldsymbol{T}(z)=\boldsymbol{G}(z) \boldsymbol{Q}(z),
$$

and every admissible controller is parameterized as

$$
\boldsymbol{C}(z)=\boldsymbol{Q}(z)(\boldsymbol{I}-\boldsymbol{G}(z) \boldsymbol{Q}(z))^{-1} .
$$

\section{B. Interpolation Conditions}

In this section we enumerate the interpolation conditions that must be satisfied by a complementary sensitivity function in order to be admissible. These conditions are key for the main result in this paper.

Define $\mathcal{C} \triangleq\left\{c_{1}, c_{2}, \ldots, c_{n_{c}}\right\}$ and $\mathcal{P} \triangleq\left\{p_{1}, p_{2}, \ldots, p_{n_{p}}\right\}$ as the sets of NMP zeros and unstable poles of $\boldsymbol{G}(z)$, respectively.

Provided Assumption 1 holds, a control loop is guaranteed to be admissible if and only if $C(z)$ yields a stable complementary sensitivity function $\boldsymbol{T}(z)$ for which the following holds (see, e.g., [14], [22]):

1) The product $\boldsymbol{E}_{\boldsymbol{c}}(z) \boldsymbol{T}(z)$ is proper and stable.

2) The interpolation condition

$$
\left.(\boldsymbol{I}-\boldsymbol{T}(z))\right|_{z=p_{i}} \boldsymbol{\mu}_{\boldsymbol{p}_{i}}=0 .
$$

holds for every $p_{i} \in \mathcal{P}$.

3) $\boldsymbol{I}-\boldsymbol{T}$ is non-singular a.e.

(We note that, if $G(\infty)=0$, then the last condition is trivially satisfied by any proper controller).

When the conditions 1) and 2) stated above are met, the controller $\boldsymbol{C}(z)$ does not cancel any NMP zero or unstable pole of $\boldsymbol{G}(z)$, and is a proper transfer matrix. Condition 3) is necessary to guarantee that the controller is also admissible.

\section{Alternative Youla Parameterization (AYP)}

Next we present a reformulation of the YP, which explicitly accounts for the interpolation conditions given in the previous section.

Lemma 1: Consider the control configuration of Figure 1 with $\boldsymbol{G}(z)$ satisfying Assumption 1 and let $\overline{\boldsymbol{T}}(z)$ be any admissible complementary sensitivity function for $\boldsymbol{G}(z)$. Then, the loop is admissible if and only if its complementary sensitivity function $\boldsymbol{T}(z)$ can be written as

$$
\boldsymbol{T}(z)=\overline{\boldsymbol{T}}(z)+\boldsymbol{E}_{\boldsymbol{c}}(z)^{-1} \boldsymbol{X}(z) \boldsymbol{E}_{\boldsymbol{p}}(z)^{-1},
$$

where $\boldsymbol{E}_{\boldsymbol{c}}(z)$ and $\boldsymbol{E}_{\boldsymbol{p}}(z)$ are zero and pole interactor matrices of $\boldsymbol{G}(z)$, and $\boldsymbol{X}(z) \in \mathcal{R H}_{\infty}$ satisfies

$$
\operatorname{det}\left\{\boldsymbol{I}-\overline{\boldsymbol{T}}-\boldsymbol{E}_{\boldsymbol{c}}(z)^{-1} \boldsymbol{X}(z) \boldsymbol{E}_{\boldsymbol{p}}(z)^{-1}\right\} \neq 0 .
$$

Proof: If $\boldsymbol{X}(z) \in \mathcal{R} \mathcal{H}_{\infty}$, then the second term in the right hand side of (16) has every NMP zero and unstable pole of $\boldsymbol{G}(z)$ as a NMP zero. Since $\overline{\boldsymbol{T}}(z)$ is an admissible complementary sensitivity function, the results follows.

The construction of (16) shows that the class of all stabilizing controllers can be characterized in terms of the knowledge of any admissible controller (equivalently, any admissible complementary sensitivity function), and the zero and pole interactor matrices. These matrices summarize all information regarding NMP zeros and unstable poles of the plant. These features are known to limit the control performance in both time and frequency domains [14]. Equation (16), moreover, makes explicit the the fact that these performance-limiting features are also fundamental for the construction of stabilizing controllers.

It is worth noting that, for stable plants, (16) takes the simple form

$$
\boldsymbol{T}(z)=\boldsymbol{E}_{\boldsymbol{c}}(z)^{-1} \boldsymbol{X}(z) .
$$

Analogously, for unstable MP plants, (16) takes the form

$$
\boldsymbol{T}(z)=\boldsymbol{I}+\boldsymbol{X}(z) \boldsymbol{E}_{\boldsymbol{p}}(z)^{-1},
$$

where $\boldsymbol{X}(z) \neq 0$. (If $X(z)=0$, then condition (17) does not hold, which is a consequence of the fact that $\overline{\boldsymbol{T}}(z)=\boldsymbol{I}$; see also Section 5.2 in [24]). However, almost all practical systems are strictly proper and, hence, they have at least one NMP zero located at infinity. Thus, the characterization of all admissible $\boldsymbol{T}(z)$ is immediate for the case of plants that are either stable or MP. In the next section, we will focus on plants that are unstable and NMP.

\section{Characterization OF AN ADMissible SENSITIVITY FUNCTION}

For stable plants, (18) describes (implicitly) any admissible controller in terms of the plant zero interactor only. This is not the case when $\boldsymbol{G}(z)$ is unstable, since both the standard and alternative formulations of the YP require prior knowledge of one admissible design. In this section, we give an explicit formula for an admissible complementary sensitivity function than can be used for controller parametrization. For future reference we define

$$
\boldsymbol{\Gamma}_{\boldsymbol{i}}(\boldsymbol{M}) \triangleq(\boldsymbol{M}-\boldsymbol{I}) \boldsymbol{\mu}_{p_{i}} \boldsymbol{\mu}_{p_{i}}{ }^{H}+\boldsymbol{I},
$$

and

$$
B_{i}(z) \triangleq\left\{\begin{array}{cc}
1 & n_{p}=1, \\
\prod_{\substack{\ell=1 \\
\ell \neq i}}^{n_{p}}\left(\frac{z-p_{\ell}}{1-z \bar{p}_{\ell}}\right)\left(\frac{1-p_{i} \bar{p}_{\ell}}{p_{i}-p_{\ell}}\right) & n_{p} \neq 1
\end{array}\right.
$$

where $i=1,2, \ldots, n_{p}$.

Lemma 2: Let $\boldsymbol{G}(z)$ satisfy Assumption 1 and denote the right directions associated with the poles of $\boldsymbol{G}(z)$ by $\left\{\boldsymbol{\mu}_{p_{1}}, \boldsymbol{\mu}_{p_{2}}, \ldots, \boldsymbol{\mu}_{p_{n_{p}}}\right\}$. Then, the complementary sensitiv- 
ity function

$$
\overline{\boldsymbol{T}}(z)=\boldsymbol{E}_{\boldsymbol{c}}(z)^{-1} \sum_{i=1}^{n_{p}} B_{i}(z) \boldsymbol{\Gamma}_{\boldsymbol{i}}\left(\boldsymbol{E}_{\boldsymbol{c}}\left(p_{i}\right)\right),
$$

is admissible.

Proof: For $\overline{\boldsymbol{T}}(z)$ to be an admissible complementary sensitivity function, it must satisfy the interpolation conditions defined in section III-B. Since $B_{i}(z)$ is stable and analytic for every zero of $\boldsymbol{G}(z)$ and for every $i$, the product

$$
\boldsymbol{E}_{\boldsymbol{c}}(z) \overline{\boldsymbol{T}}(z)=\sum_{i=1}^{n_{p}} B_{i}(z) \boldsymbol{\Gamma}_{\boldsymbol{i}}\left(\boldsymbol{E}_{\boldsymbol{c}}\left(p_{i}\right)\right),
$$

is a stable and proper transfer matrix.

For the interpolation condition (15), we first note that

$$
B_{i}\left(p_{j}\right)=\delta(i-j),
$$

where $\delta(t)$ denotes the Kronecker's delta. Thus,

$$
\left(\boldsymbol{I}-\overline{\boldsymbol{T}}\left(p_{j}\right)\right) \boldsymbol{\mu}_{\boldsymbol{p}_{\boldsymbol{j}}}=\left(\boldsymbol{I}-\boldsymbol{E}_{\boldsymbol{c}}\left(p_{j}\right)^{-1} \boldsymbol{\Gamma}_{\boldsymbol{j}}\left(\boldsymbol{E}_{\boldsymbol{c}}\left(p_{i}\right)\right)\right) \boldsymbol{\mu}_{\boldsymbol{p}_{\boldsymbol{j}}} .
$$

From (20) it follows that

$$
\Gamma_{j}(M) \mu_{p_{j}}=M \mu_{p_{j}},
$$

then the right side of (25) vanishes. Since $p_{j}$ is an arbitrary unstable pole of $\boldsymbol{G}(z)$, condition (15) holds.

Lemma 2 states that, in the case where all the unstable poles of $\boldsymbol{G}(z)$ are simple, an admissible complementary sensitivity function can be constructed without the use of any design method. Only dynamical features of $\boldsymbol{G}(z)$, namely NMP zeros, unstable poles and their respective directions, are employed. If we consider a single-input single-output (SISO) plant $\boldsymbol{G}(z)$, then the expression for $\overline{\boldsymbol{T}}(z)$ simplifies to

$$
\overline{\boldsymbol{T}}(z)=\boldsymbol{E}_{\boldsymbol{c}}(z)^{-1} \sum_{i=1}^{n_{p}} B_{i}(z) \boldsymbol{E}_{\boldsymbol{c}}\left(p_{i}\right)
$$

It is worth noting that the controller associated with $\overline{\boldsymbol{T}}(z)$ is given by (see (7))

$$
\begin{gathered}
\overline{\boldsymbol{C}}(z)=\boldsymbol{G}(z)^{-1}(\boldsymbol{I}-\overline{\boldsymbol{T}}(z))^{-1} \overline{\boldsymbol{T}}(z) . \\
\text { V. EXAMPLES }
\end{gathered}
$$

This section presents two examples to illustrate the results in this paper.

\section{A. SISO plant}

Let $G(z)$ be a scalar LTI plant given by

$$
G(z)=\frac{g_{0}(z-c)}{\left(z-p_{1}\right)\left(z-p_{2}\right)}
$$

where $g_{0} \in \mathbb{R},|c|<1$ and both poles are unstable, real and distinct. Then, the zero interactor is given by

$$
E_{c}(z)=z \text {. }
$$

Using (27), we have that an admissible complementary sensitivity function is given by

$\bar{T}(z)=\frac{1}{z}\left[\left(\frac{z-p_{2}}{1-p_{2} z}\right) p_{1}-\left(\frac{z-p_{1}}{1-p_{1} z}\right) p_{2}\right]\left(\frac{1-p_{1} p_{2}}{p_{1}-p_{2}}\right)$.

The interpolation conditions are easily verified.

The associated controller (see (28)) is given by

$$
\bar{C}=\frac{\left(p_{1} p_{2}-1\right)\left(\left(p_{1}+p_{2}\right) z-p_{1} p_{2}-1\right)}{g_{0} p_{1} p_{2}(z-c)},
$$

which is biproper and cancels the MP zero of $G(z)$.

\section{B. MIMO plant}

Let $G(z)$ be a MIMO LTI plant given by

$$
\boldsymbol{G}(z)=\left[\begin{array}{cc}
\frac{1}{(z-3)} & -\frac{z}{(z-2)(z-3)} \\
0 & \frac{1}{(z-2)}
\end{array}\right],
$$

which is strictly proper, has two simple unstable poles at $z=p_{1}=2$ and $z=p_{2}=3$ and two NMP zeros at infinity. The unitary zero interactor for $\boldsymbol{G}(z)$ is

$$
\boldsymbol{E}_{\boldsymbol{c}}(z)=\operatorname{diag}\{z, z\}
$$

The unitary right directions for the unstable poles are

$$
\boldsymbol{\mu}_{\boldsymbol{p}_{1}}=\left[\begin{array}{c}
0.8944 \\
0.4472
\end{array}\right], \quad \boldsymbol{\mu}_{\boldsymbol{p}_{2}}=\left[\begin{array}{l}
1 \\
0
\end{array}\right] \text {. }
$$

With this, using (22) we obtain an admissible complementary sensitivity function

$$
\begin{gathered}
\overline{\boldsymbol{T}}(z)=\boldsymbol{E}_{\boldsymbol{c}}(z)^{-1} \sum_{i=1}^{2} B_{i}(z) \boldsymbol{\Gamma}_{\boldsymbol{i}}\left(\boldsymbol{E}_{\boldsymbol{c}}\left(p_{i}\right)\right) \\
=\left[\begin{array}{cc}
T_{11}(z) & T_{12}(z) \\
T_{21}(z) & T_{22}(z)
\end{array}\right],
\end{gathered}
$$

where

$$
\begin{aligned}
& T_{11}(z)=\frac{4.5(z-1.481)(z-0.07505)}{z(z-0.5)(z-0.3333)}, \\
& T_{12}(z)=-\frac{0.66667(z-3)}{z(z-0.3333)} \\
& T_{21}(z)=-\frac{0.66667(z-3)}{z(z-0.3333)} \\
& T_{22}(z)=\frac{0.5(z+3.174)(z-0.8403)}{z(z-0.5)(z-0.3333)} .
\end{aligned}
$$

VI. CONCLUSIONS

In this paper we have proposed a methodology to explicitly construct an admissible complementary sensitivity function using only fundamental dynamical features of an LTI plant. These features are the unstable poles and NMP zeros of the plant, and their directions in the MIMO case. As a byproduct, 
it has been shown that the information contained in the stable and MP part of a given plant is irrelevant when constructing an admissible complementary sensitivity function for it. We note that the construction described here is non-unique and different expressions can be obtained using simple variations of the ideas contained herein.

\section{ACKNOWLEDGEMENTS}

The second author gratefully acknowledges the support of Science Foundation Ireland (SFI) under Research Professor Award 03/RP1/I382. The other authors are grateful for the support provided by UTFSM and Fondecyt-Chile through grant 1080274 .

\section{REFERENCES}

[1] M. Morari and E. Zafiriou, Robust process control. Englewood Cliffs, New Jersey: Prentice Hall Inc., 1989.

[2] S. Skogestad and I. Postlethwaite, Multivariable Feedback Control: Analysis and Design. New York: Wiley, 2000.

[3] G. C. Goodwin, S. Graebe, and M. E. Salgado, Control System Design. New Jersey: Prentice Hall, 2001.

[4] D. Youla, H. Jabr, and J. Bongiorno, "Modern Wiener-Hopf design of optimal controllers. Part I," IEEE Transactions on Automatic Control, vol. 21, no. 2, pp. 3-13, 1976.

[5] —_, "Modern Wiener-Hopf design of optimal controllers. Part II: The multivariable case," IEEE Transactions on Automatic Control, vol. 21 , no. 3, pp. 319-338, 1976.

[6] D. Youla and J. Bongiorno, "A feedback theory of two-degree-offreedom optimal wiener-hopf design," IEEE Transaction on Automatic Control, vol. 30, pp. 652-665, 1985.

[7] B. Francis, J. W. Helton, and G. Zames, "H户 $\mathcal{H}_{\infty}-$ optimal feedback controllers for linear multivariable systems," IEEE Transaction on Automatic Control, vol. 29, pp. 888-900, 1984.

[8] V. Kucera, "The $\mathcal{H}_{2}$ control problem: a general transfer-function solution," International Journal of Control, vol. 80, pp. 800-815, 2007.

[9] J. Doyle, K. Glover, P. Khargonekar, and B. Francis, "State-space solutions to standard $\mathrm{H}_{2}$ and $\mathrm{H}_{\infty}$ control problems," IEEE Transactions on Automatic Control, vol. AC-34, no. 8, pp. 831-847, 1989.
[10] H. Rotstein and M. Sznaier, "An exact solution to general four-block discrete-time mixed $\mathcal{H}_{2} / \mathcal{H}_{\infty}$ problems via convex optimization," IEEE Transactions on Automatic Control, vol. 43, no. 10, pp. 1475$1480,1998$.

[11] W. Zhang, F. Allgöwer, and T. Liu, "Controller parameterization for SISO and MIMO plants with time delay," Systems \& Control Letters, vol. 55, pp. 794-802, 2006.

[12] M. Salgado and E. Silva, "Robustness issues in $\mathcal{H}_{2}$ optimal control of unstable plants," Systems \& Control Letters, vol. 55, no. 2, pp. 124 - 131, February 2006

[13] A. Peters, M. Salgado, and E. Silva, "Performance bounds in linear control of unstable MIMO systems with pole location constraint," Systems \& Control Letters, vol. 57, pp. 392-399, 2008.

[14] M. M. Seron, J. H. Braslavsky, and G. C. Goodwin, Fundamental Limitations in Filtering and Control. London: Springer Verlag, 1997.

[15] O. Toker, L. Chen, and L. Qiu, "Tracking performance limitations in LTI multivariable discrete-time systems," IEEE Transactions on Circuits and Systems-Part I: Fundamental Theory and Applications, vol. 49, no. 5, pp. 657-670, May 2002.

[16] A. Peters and M. Salgado, "Performance bounds in H-infinity optimal control for stable SISO plants with arbitrary relative degree," IEEE Transactions on Automatic Control (to appear), 2009.

[17] T. Kailath, Linear Systems. Englewood Cliffs, New Jersey: PrenticeHall, 1980.

[18] E. Silva and M. Salgado, "Performance bounds for feedback control of nonminimum-phase MIMO systems with arbitrary delay structure," IEE Proceedings - Control Theory and Applications, vol. 152, no. 2, pp. 211-219, March 2005.

[19] D. Bernstein, Matrix Mathematics. Princeton University Press, 2005.

[20] J. M. Maciejowski, Multivariable Fedback Design. Wokingham, England: Addison Wesley, 1989.

[21] A. MacFarlane and N. Karcanias, "Poles and zeros of linear multivariable systems: a survey of the algebraic, geometric and complex variable theory," International Journal of Control, vol. 24, no. 1, pp. 33-74, 1976.

[22] K. Zhou, J. Doyle, and K. Glover, Robust and optimal control. Englewood Cliffs, New Jersey: Prentice Hall, 1996.

[23] B. Francis, $A$ Course on $H_{\infty}$ Control Theory, ser. Lecture Notes in Control and Information Sciences. New York: Springer Verlag, 1987, vol. 8 .

[24] M. Vidyasagar, Control systems synthesis: A Factorization Approach. Cambridge, Mass: MIT Press, 1985. 